\title{
John Dewey y el Desarrollo de la Pedagogía Rusa antes de 1930 - Informe sobre una recepción olvidada
}

\author{
John Dewey and the Development of Education \\ in Russia before 1930 - Report on \\ a Forgotten Reception
}

\section{John Dewey et le Développement de l'Éducation en Russie avant 1930 - Rapport sur un accueil oublié}

Dedicado a la memoria de mi padre, Jurij Surenovič Mchitarjan

\section{Irina Mchitarjan}

Ernst-Moritz-Arndt Universität Greifswald, Alemania

\section{RESUMEN}

El presente artículo explora la problemática de la recepción de John Dewey (1859-1952) en Rusia entre los años 1900 y 1930, así como también su influencia en la discusión pedagógica en ese país. Los principales resultados que hemos obtenido son los siguientes: tanto antes como después de la Revolución de Octubre (1917), Dewey tuvo una gran relevancia en el desarrollo del sistema escolar ruso. El rechazo final de la obra de Dewey hacia finales de la década de 1920 se produjo no por causas pedagógicas, sino por motivos políticos e ideológicos. ${ }^{1}$

Descriptores: Dewey-recepción; Rusia; educación progresivo; historia de la educación.

\section{ABSTRACT}

This article explores the kinds of response John Dewey (1859-1952) received in Russia between 1900 and 1930, and the impact he had on the educational debate there. The study's main findings are: Both before and after the Socialist October Revolution of 1917, Dewey had a significant impact on the development of the Russian school system. The ultimate rejection 
of Dewey's pedagogy towards the end of the 1920s was not due to educational but to political and ideological reasons.

Key words: Dewey; Dewey-reception; Russia; progressive education; history of education.

RÉSUMÉ

Cet article explore les sortes de réponses que John Dewey (1859-1952) reçut en Russie entre 1900 et 1930, et l'impact qu'il eut sur le débat éducatif. Les principales conclusions de l'étude sont : Autant avant qu'après la Révolution socialiste d'octobre 1917, Dewey eut un impact significatif sur le développement du système scolaire socialiste. Le rejet ultime de la pédagogie de Dewey vers la fin du 20e siècle ne fut pas à cause de raisons éducatives mais bien politiques et idéologiques.

Mots clés : Dewey; Dewey-réception; Russie; l'éducation progressif; d'histoire de l'éducation.

Jors

OHN DEWEY (1859-1952) "es uno de los pocos pedagogos del siglo XX cuya obra llegó a tener influencia a nivel mundial, tanto en la teoría como en la práctica" (Klafki 1978, p. 781). La obra de Dewey fue objeto de una intensa recepción en Rusia - entre otros países - en especial en el período comprendido entre 1900 y 1930. Sin embargo, este recepción de la pedagogía de Dewey en Rusia fue hasta ahora (con ciertas excepciones, cf. Komarovskij 1926) un terreno en gran medida inexplorado. El presente trabajo intenta ofrecer una nueva apreciación de esta parte olvidada de la historia de las ciencias de la educación en Rusia. El interrogante central es: ¿cuál fue la recepción que se brindó a la obra de Dewey en Rusia entre los años 1900 y 1930, y de qué manera influyó ésta en la discuión pedagógica del país? Es mi intención, en primer lugar, establecer las razones y los condicionamientos que llevaron a la recepción de Dewey en Rusia; intentaré luego una reconstrucción de la recepción desde la cronología y los contenidos de la misma; por último, he de plantear la cuestión de la significación y función que le cupo a la recepción de Dewey en el proceso de reforma educativa en Rusia. ${ }^{2}$

Para comprender la historia de la recepción de Dewey en Rusia durante el primer tercio del siglo XX, es necesario tener una noción básica del trasfondo histórico - en especial, del trasfondo político-escolar - contra el cual ésta tuvo lugar. Comenzaremos, por ello, con una suscinta descripción del contexto histórico. Hemos de dedicar la segunda parte de nuestro trabajo a los aspectos esenciales del la recepción de Dewey.

\section{Contexto histórico y social}

1) El desarrollo del sistema escolar ruso entre 1900 y 1930 — vale decir, en el período de recepción que nos proponemos explorar - se vio fuertemente influido por el desarrollo general de los fenómenos sociales en el país. Como principales cesuras históricas - que hicieron sentir su efecto tanto en el desarrollo de la escuela como en la recepción de Dewey - pueden considerarse la revolución democrático-burguesa de 1905, la revolución socialista de octubre de 1917 y el comienzo del primer Plan Quinquenal en 1928. 
A principios del siglo XX imperaba todavía en Rusia una monarquía absoluta que se caracterizaba por una política poco liberal y represiva frente a la crítica pública. La revolución democrático-burguesa de 1905 señaló la transición de la monarquía absoluta a una constitucional y trajo aparejadas ciertas libertades democráticas. Esta situación condujo, entre otras cosas, al surgimiento de un público pedagógico de carácter crítico, con fuertes tendencias hacia la reforma educativa (escuelas reformadas, publicaciones especializadas, congresos pedagógicos, etc.).

La revolución socialista de octubre de 1917, que llevó a la fundación del estado soviético, trajo consigo una transformación radical en la política educativa rusa. Los pedagogos soviéticos, si bien se esforzaron por resolver varios problemas de la escuela, excluyeron del debate (y de la realización práctica) toda iniciativa de reforma que resultase ideológicamente sospechosa. Las ideas reformistas - aun aquellas que provenían del extranjero - debían amoldarse al ideal de educación soviético. Sin embargo, pese a todos los controles impuestos desde el estado, puede verse en la década de 1920 (hasta el comienzo del Plan Quinquenal en 1928) un período de intensa experimentación pedagógica en la Rusia soviética.

Tras el comienzo del primer Plan Quinquenal en 1928 - cuyo propósito era el de lograr una industrialización acelerada en Rusia - se tenía la idea de promover en el sistema educativo medidas que favorecieran "el crecimiento cultural de las grandes masas obreras" (escolaridad obligatoria, erradicación del analfabetismo, educación técnico-profesional de masas), así como también la formación de especialistas cualificados y científicos (KPSS v rezolucijach s-ezdov, konferencij i plenumov CK 1954, p. 465). Estas medidas iban de la mano con una "intensificación de la lucha de clases" y un incremento del control del partido (también en el ámbito escolar). En virtud de la resolución del Comité Central "sobre la escuela elemental y media," aprobada el 5 de septiembre de 1931, se prohibió "terminantemente la experimentación en las escuelas de instrucción general” (Froese 1963, p. 196). También se prohibió, de allí en más, cualquier referencia al legado de la pedagogía reformista rusa de la época prerrevolucionaria o a modelos de reforma tomados del extranjero.

2) El sistema escolar ruso de finales de siglo XIX estaba segmentado en dos subsistemas: una educación “inferior" y otra "superior." En lo que respecta a la educación "inferior," no existía en Rusia a comienzos del siglo XX una escolaridad pbligatoria reglamentada ni tampoco la cantidad de escuelas necesaria para hacerla posible. En 1897, sólo una cuarta parte de la población sabía leer y escribir (Johnston 1969, p. 173s.). A las escuelas "superiores" rusas asistía, a finales del siglo XIX, sólo un 0,13\% de la población (Johnston 1969, p. 196). El acceso a la educación superior se encontraba estrictamente limitado por ley (cf. Konstantinov 1947, p. 48). Por estas razones, los temas que dominaban la discusión pedagógica en Rusia a finales del siglo XIX eran la ampliación de la red de escuelas primarias y la introducción de la escolaridad obligatoria, la abolición de la división entre las escuelas de bachillerato tradicionales y las demás secundarias, y la conexión de la escuela elemental con las instituciones "superiores" dentro de un sistema escolar integrado. 
3) La apreciación que los principales actores en el terreno de la política escolar rusa entre 1900 y 1917 - los "pedagogos estatales" y las fuerzas reformistas — hacían de estos problemas era muy diversa. Mientras que la intención de los "pedagogos estatales" - quienes ejercían sus funciones en organismos vinculados con la iglesia y el estado - era la de preservar el sistema en un nivel de subdesarrollo, las fuerzas reformistas (si bien con distintas orientaciones pedagógicas) apostaban, con una intensidad cada vez mayor, a la introducción de la escolaridad obligatoria general y al establecimiento de un sistema educativo común, homogéneo y reformado en su pedagogía. Más allá de estos reclamos conjuntos, cada una de las fuerzas reformistas trabajaba en sis propios proyectos de reforma (cf., en mayor detalle, Mchitarjan 1998). En este marco, fueron fundamentalmente dos importantes corrientes reformistas de la época — la de la "educación libre" y la de la "educación realista" — las que actuaron en la recepción de la pedagogía de Dewey antes de 1917.

La corriente de la "educación libre" estaba inspirada en las enseńanzas de L. Tolstoj (1828-1910), a quien por lo general se considera fundador de la pedagogía reformista rusa. Sus discípulos (Vencel, Durylin, Gorbunov-Posadov, Klečkovskij, Čechov, Šackij y Kajdanova, Zelenko, Šleger y Krupskaja) favorecían la autonomía escolar, es decir, la liberación de la escuela del control administrativo del estado y su transferencia a organismos comunales autoadministrados; promovían también el derecho de cada escuela de conformar de manera autónoma su praxis pedagógica (cf., por ejemplo, Vencel 1908; Čechov 1907; Otčet o dejatel'nosti pedagogičeskogo obščestva 1899).

Por su parte, los proyectos de los pedagogos "realistas" (Obuchov, Sokolov, Levitin, Ignat'ev, Rumjancev, Znamenskij, entre otros) surgieron contra el telón de fondo de la transformación industrial que tuvo inicio hacia 1890. Pretendían, ante todo, consolidar el principio del trabajo en las escuelas elementales y extender la formación vocacional. Su objetivo era dar prioridad a una educación realista, científica y orientada a la práctica (cf. Medynskij 1938, pp. 377ss., 381; véase además "Reforma Srednej Školy” 1915, p. 78-85).

4) En la década de 1920, las autoridades soviéticas impusieron a la discusión pedagógica límites aún más estrechos de lo que lo había hecho el régimen zarista (cf. Kapterev 1918, pp. 147-149). La anterior multiplicidad de corrientes pedagógicas, que contaban con independencia en sus organizaciones, publicaciones y experimentos escolares, cayó víctima del control estatal. A partir de entonces, toda posición crítica a la pedagogía marxista del estado pasó a ser considerada "contrarrevolucionaria."

Sin embargo, el campo dominante en la nueva pedagogía estatal tampoco era — ni en lo pedagógico ni en lo político - del todo homogéneo y contenía ciertos elementos liberales. Los partidarios más firmes de la doctrina marxista-leninista (como Pinkevič, Šulgin y Kamenev) se orientaban, en lo político, hacia la idea de la lucha de clases, la dictadura del proletariado y el combate activo contra el enemigo de clase; en lo pedagógico, intenetaban aplicar activamente estos objetivos del estado en la escuela. Se declaraban de manera cada vez más frecuente-en especial, en la segunda mitad de la década de 1920 — en contra de las influencias en la Rusia 
soviética de corrientes pedagógicas extranjeras y de la pedagogía reformista rusa de la época zarista, a la que tildaban de liberal y "pequeñoburguesa" (cf. Pinkevič 1925, 1930; Šulgin 1925b; Kamenev 1925).

La pedagogía liberal soviética (Šackij, Blonskij, Fortunatov, Gorbunov-Posadov, Vencel, Čechov, Kapterev y Levitin) se nutría en su mayoría de las filas de la pedagogía reformista prerrevolucionaria. Sus exponentes decidieron, tras poco pensarlo, colaborar con las autoridades soviéticas. Proponían la adopción crítica tanto del legado de la pedagogía nacional prerrevolucionaria como la pedagogía reformista extranjera y - en contados casos - apoaban una realización no dogmática de la doctrina marxista. Abogaban por la igualdad de derechos para los niños de todas las clases sociales y se oponían a toda forma de violencia, incluso si ésta provenía del estado (cf., p.ej., Vencel 1916/1917, pp. 1-8 y 1918, pp. 13-18). En vista de tal actividad, no es de extrañar que los marxistas fieles a los lineamientos partidarios criticaran frecuentemente - en especial, hacia finales de la década de 1920 — a los pedagogos liberales por su falta de decisión y radicalidad en la consecución de los objetivos políticos del nuevo estado (cf., p.ej., Pinkevič 1925, pp. 84-88).

Entre ambos grupos se encontraba una serie de pedagogos soviéticos (p.ej., el ministro de Educación A. V. Lunačarskij y N. K. Krupskaja, funcionaria del Ministerio) que representaban una especie de posición intermedia, a proponer una implementación total, pero no dogmática, de la doctrina marxista-leninista. Eran lo suficientemente pragmáticos para constatar que resultaba imposible construir un nuevo sistema educativo en la Rusia soviética sin valerse de modelos de orientación y que los modelos extranjeros de educación "nueva" o "progresista" (pese a su carácter, en última instancia, "burgués") podían redundar en beneficio de tal proceso. El mejor ejemplo de ello es la intensidad con que se llevó a cabo la recepción de la obra de Dewey en Rusia soviértica durante la década de 1920.

\section{Principales líneas de la recepción de Dewey en Rusia (1900-1930)}

\subsection{La recepción de Dewey antes de 1917}

La recepción de la pedagogía de Dewey en Rusia comenzó en 1907, en el contexto del fortalecimiento del movimiento ruso de reforma pedagógica que se verificó tras la revolución burguesa de 1905. En esa época se dio en Rusia una búsqueda particularmente intensa de modelos de reforma escolar. Esta circunstancia favoreció especialmente la recepción de proyectos de reforma extranjeros (cf. Vencel 1906; Čechov 1907; Šackij 1907/1908; Šackij/Zelenko/Kazimirov 1908; Mchitarjan 1998). Al mismo tiempo, Dewey presentaba un concepto pedagógico que concitaba el interés de distintas tendencias de la pedagogía reformista. Sin embargo, su enfoque fue considerado en esta época como uno más entre otros muchos modelos de reforma.

En el período comprendido entre 1907 y 1917, pueden encontrarse no menos de 16 referencias a la pedagogía de Dewey en las fuentes que hemos estudiado (trabajos de pedagogos rusos, diarios de sesiones, actas de congresos, etc.). Éstas van 
desde la publicación de obras de Dewey en traducción rusa hasta breves menciones de su enfoque en las discusiones mantenidas en congresos. La recepción de Dewey en Rusia fue, antes de 1917, decididamente escasa, sobre todo si se la compara con la de G. Kerschensteiner, el pedagogo occidental de mayor popularidad en la Rusia prerrevolucionaria (cf. Mchitarjan 1998). Mientras que hasta 1917 se publicaron en traducción rusa no menos de 13 libros y 2 artículos de Kerschensteiner, sólo se tradujeron al ruso en el mismo período tres libros de Dewey (Škola i obščestvo [The School and Society] (1907); Psichologia i pedagogika myšlenija [How we think] (1915) y Školy buduščego [Schools of Tomorrow] (1916/1917)). Mientras que las ideas de Kerschensteiner dieron lugar a alrededor de cincuenta reacciones relativamente extensas por parte de autores rusos, en el caso de Dewey sólo hubo, en el mismo período, trece reacciones. Y éstas sólo pocas veces tienen el carácter de discusiones críticas y abarcativas de su enfoque pedagógico: en su mayor parte no pasan de breves menciones, referencias o remisiones a su obra. Tanto más sorprendente resulta entonces que - poco antes de la Revolución de Octubre - Dewey haya llegado a tener un papel tan importante como el de Kerschensteiner (si no más importante que éste) en el desarrollo del sistema educativo ruso. Esta situación es la que explicaremos en mayor detalle en la sección siguiente.

\subsection{Los exponentes de la "educación libre" y la recepción de Dewey (1905-1917)}

La recepción de Dewey anterior a 1917 se caracterizó por el hecho de que los exponentes de la "educación libre" y de la "educación realista" inclinaban su interés hacia aspectos diversos en las enseñanzas de Dewey. Los partidarios de la "educación libre” se interesaban particularmente por el reclamo de Dewey de una organización del proceso pedagógico centrada en el niño, del aprendizaje a través de la experiencia y por su concepción amplia de la edicación por medio del trabajo. El caso es que, con la educación por medio del trabajo, los discípulos de Tolstoj no pretendían solucionar los problemas económicos de su tiempo, sino pormover una formación armónica del ser humano. No les interesaba el trabajo en el sentido "político-económico," sino en el sentido "ético" y "pedagógico" (cf., p.ej., Vencel 1908; Šackij/Zelenko/Kazimirov 1908). Y en Dewey veían corroborados estos puntos de vista. Por otra parte, Dewey llamó la atención de los partidarios de la "educación libre" no sólo por sus reclamos programáticos, sino también por los resultados obtenidos en su implementación práctica.

Por ejemplo, K. Vencel consideraba la escuela experimental fundada por Dewey en la universidad de Chicago como una posible "escuela ideal del futuro" (cf. Vencel 1908/1909). Según lo entendía Vencel, esta escuela ideal debía carecer de todo tipo de coerción y castigo; debía ser una escuela que organizara su enseñanza a partir del interés del niño, una escuela que lograra, por medio de la aplicación direccionada del trabajo, un desarrollo integral del niño. Para Vencel, como partidario de la "educación libre," revestía particular importancia el el hecho de que Dewey, en su concepción de la escuela del trabajo, se propusiera como objetivo no tanto el desarrollo de habilidades manuales concretas, sino más bien una educación y una formación 
integral de los jóvenes (Vencel en ACARE F. 23, op.1, ed. chr. 13).

Las teorías de Dewey llamaron también la atención de otro representante de la "educación libre," Stanislav Šackij, por "su insistencia en comprobar las ideas en la vida real" y su "perspicaz análisis del interés infantil” (Šackij 1928 en Rüttenauer/ Schiff 1970, p. 386). Ya en 1910, Šackij reconoció "cierto grado de influencia de las ideas de Dewey en el desarrollo de [sus propias] concepciones pedagógicas" (id., p. 386). Con ello, Šackij da prueba explícita de aquello que nos permiten suponer, con bastante grado de certeza, los testimonios indirectos: la pedagogía de Dewey fue una de las influencias que pesaron sobre los proyectos de reforma de los principales educadores refomistas de la Rusia prerrevolucionaria.

Además, la perspectiva de los discípulos de Tolstoj coincidía con la recepción de Dewey por parte de los autores alemanes (F. Gansberg, A. Pabst), cuyos trabajos se publicaban en el periódico "La Educación Libre." Es decir, los autores alemanes se remitían a las concepciones de Dewey en exactamente las mismas temáticas en las que lo hacían los discípulos de Tolstoj (cf. Gansberg 1907/1908, pp. 73-92 y 1915, pp. 21-26; Pabst 1909/1910, pp. 63-88).

Puede decirse, en resumen, que la recepción de Dewey por parte del movimiento de la "educación libre" comportaba una amplia variedad de temas. Pero, ante todo, se la hacía corresponder con los propios proyectos de reforma de sus receptores rusos. Eran los proyectos de reforma de los discípulos de Tolstoj los que determinaban tanto el foco temático del recepción como la evaluación de la relevancia pedagógica de la obra de Dewey.

\subsection{Los seguidores de la "educación realista" y la recepción de Dewey (1905-1917)}

Mientras que los representantes de la "educación libre" hacían una recepción de Dewey de carácter relativamente amplio en lo temático, los seguidores de la "educación realista" sólo se interesaban por un aspecto en particular de su enfoque: el concepto de la formación por medio del trabajo. Así y todo, los pedagogos "realistas" observaban el concepto desde una perspectiva distinta de la de los discípulos de Tolstoj. Mientras que los partidarios de la "educación libre" se interesaban por la función pedagógica del principio del trabajo, los seguidores de la educación "realista" ponían el acento en su aporte a la cualificación de la mano de obra "moderna."

Es desde esta perspectiva que P. Sokolov (1913) comenta la crítica de Dewey a la "vieja" escuela. Según Sokolov, Dewey había demostrado que la "vieja" escuela" no contribuía ni al desarrollo de la individualidad del alumno ni al de su vida pública; en tal tipo de escuela, la enseñanza se encuentra aislada de la realidad cotidiana y las experiencias del nińo, de modo que el conocimiento transmitido de tal manera no es aprovechable para la sociedad industrial moderna (cf. Sokolov 1913, p. 589s.). Como alternativa, Sokolov propone la escuela del trabajo de Dewey. Otro de los principales exponentes de la educación "realista," S. A. Levitin, se expresaba en términos similares: la nueva escuela debía ser una escuela del trabajo, tal como — según Levitin — ya se la había implementado en los Estados Unidos, con Dewey como su representante 
más sobresaliente (Levitin 1912/1913, p. 27ss.; 1916).

En el Primer Congreso Panruso de Educación Popular, que tuvo lugar en San Petersburgo entre el 22 de diciembre de 1913 y el 3 de enero de 1914, el tópico de la "escuela del trabajo" constituyó - junto con las problemáticas de la escolaridad obligatoria y la escuela integrada - uno de los temas centrales de discusión. ${ }^{3}$ Se presentaron numerosas ponencias acerca de "la escuela y la educación del trabajo." La tesis básica de casi todas ellas era la siguiente: la escuela del trabajo era la mejor solución para los problemas de la época, tanto pedagógicos como económicos (cf. Levitin 1914/1915). ${ }^{4}$ Para fundamentar esta tesis, algunos pedagogos "realistas" (p.ej., Kasatkin, Levitin y Repin) hicieron referencia, entre otros autores, también a Dewey y su concepción de una formación por medio del trabajo, citando obras de Dewey y alegando que gracias a la implementación del principio del trabajo en las escuelas norteamericanas, se habían logrado en los Estados Unidos importantes avances pedagógicos (cf. "Doklady" 1915, pp. 46 y 51). Mediante el principio del trabajo - se argumentaba - la escuela norteamericana no sólo había mejorado la enseñanza, sino que también había conseguido logros considerables en la educación social de los jóvenes (cf. "Doklady" 1915, p. 78). Y, en consecuencia, se reclamaba: "También nuestra escuela debe ir en la misma dirección, es decir, debe vivificar el proceso de formación y educación por medio del trabajo práctico, tal como lo ha hecho la escuela norteamericana ("Doklady" 1915, p. 78).

Estos argumentos de los pedagogos "realistas" - sustentados por la autoridad de Dewey - tenían por destinatarios a dos grupos: los maestros rusos y los pedagogos "estatales." Mientras que se esperaba ganar con ellos a los maestros rusos para la idea de la escuela del trabajo, su propósito era también forzar a los pedagogos "estatales" a abandonar su postura contraria a la reforma (cf. "Doklady" 1915; Levitin 1914/1915; 1915). La estrategia dio resultado: el Primer Congreso Panruso de Educación Popular declaró el principio del trabajo como "elemento necesario en la escuela de formación general" y como lineamiento que estaba llamado a "reformar la organización interna de la escuela." ${ }^{5}$ Así, el principio del trabajo fue reconocido públicamente como uno de los elementos centrales de la reforma de las escuelas comunes, incorporándose a los aspectos fundamentales del movimiento de reforma escolar ruso. Y es seguro que las referencias a Dewey tuvieron su parte en este éxito de los pedagogos "realistas." Si bien las conclusiones del congreso no eran de carácter vinculante, la idea de la escuela del trabajo pudo, al menos, ser puesta en práctica por los maestros.

\subsection{Los planes de reforma del P. N. Ignat'ev, ministro de Educación Pública (1915)}

En 1915 puede hablarse ya de una influencia de Dewey sobre la política educativa oficial rusa. Fue en ese momento que las ideas reformistas de Dewey encontraron eco en los proyectos de reforma escolar del Ministerio de Educación Pública. Ello sólo se hizo posible mediante el nombramiento de un partidario de la educación “realista," el conde Pavel Nikolaevič Ignat' ev, ${ }^{6}$ al frente del Ministerio de Educación ruso. Desde esa posición, Ignat'ev intentó poner en marcha una reforma integral del 
sistema educativo, tomando a Dewey como priera fuente de inspiración.

Los planes del nuevo ministro se proponían superar el atraso del sistema escolar ruso y colocarlo a un nivel comparable con el de Europa occidental. La escuela reformada sería una escuela integrada con lugar para la enseńanza práctica. En los nuevos currículos se concedía un amplio espacio a las clases prácticas de Física, Química y Biología, así como también al Dibujo Técnico y la Educación Física. Se tenía asimismo en consideración el valor pedagógico de las actividades manuales, y se proponía su paulatina incorporación como asignatura obligatoria (cf. Konstantinov 1947, p. 188). Ignat'ev atribuía, además, una gran importancia a la formación técnico-vocacional, fundando, con el fin de promoverla, el "Consejo Ruso de Formación Vocacional" ("Reforma srednej školy" 1915).

Testimonio de la influencia de Dewey en los planes de reforma del Ministerio de Educación ruso lo brindan los datos siguietes: el nuevo ministro había estudiado, ya antes de 1915, uno de los trabajos más importantes de Dewey, el libro titulado The School and Society, y había visto - según su propio comentario — en la filosofía y la pedagogía de Dewey el fundamento teórico para la reforma educativa que él mismo tenía en mente desde hacía un tiempo. Llamaron su atención, en especial, las ideas de Dewey respecto del aprendizaje práctico, la educación orientada hacia la realidad y la escuela como "parte del entorno del niño" (cf. Ignat'ev en Ignat'ev/Odinetz/ Novgorotsev 1929, p. xxii). Escribe Ignat'ev que él y sus colaboradores encontraron en el libro de Dewey "la mayor ayuda y el mayor incentivo ... para llevar a la práctica las mismas ideas en Rusia" (Ignat'ev en Ignat'ev/Odinetz/Novgorotsev 1929, p. xxii). Se dio, además, el caso de que algunos de los principales pedagogos reformistas - en particular, los receptores activos de Dewey (como Levitin) - participaran en la discusión de las reformas propuestas por el Ministerio (cf. "Reforma srednej školy" 1915, p. 78-85).

Los planes de Ignat'ev representaban un transformación radical en la "pedagogía estatal." Resultaban adecuados para solucionar los problemas más graves que aquejaban al sistema escolar ruso y ponerlo en el nivel del desarrollo económico y social de la época. Por otra parte, también hacían lugar a los principales reclamos de los pedagogos reformistas: escuela integrada y escuela del trabajo. Tanto en la elaboración de los planes cuanto en sus contenidos concretos, la influencia de Dewey parece haber sido considerable. En general, en la Rusia de 1915 podía tenerse la impresión de que las ideas reformistas de Dewey (junto con las de Kerschensteiner, cf. Mchitarjan 1998) habrían de desempeñar un papel decisivo en el desarrollo futuro del sistema escolar ruso. Sin embargo, estas ideas sólo pudieron ser llevadas a la práctica de modo parcial: la implementación de los planes de Ignat'ev se vio obstaculizada por la Primera Guerra Mundial y por la revolución socialista de octubre de 1917. Pero ello no significó el fin de la recepción de Dewey en Rusia.

\subsection{La recepción de Dewey después de 1917}

En la Rusia soviética, la recepción de Dewey experimentó un enorme auge. Se publicaron, en parte con varias reimpresiones, obras de Dewey tales como: Vvedenie v 
filosofiju vospitanija [Democracy and Education: An Introduction to the Philosophy od Education] (1921), Školy buduščego [Schools of Tomorrow] (1922d), Škola $i$ obščestvo [The School and Society] (1922b, 1924, 1925), Škola i rebenok [The Child and the Curriculum] (1922c, 1923) y Psichologija i pedagogika myšlenija [How we think] (1922a). ${ }^{7}$ Asimismo su enfoque de la pedagogía fue tratado (en forma exclusiva o dentro de un contexto temático más amplio) en más de cuarenta artículos de autores rusos. Los principales "pedagogos estatales” de la Rusia soviética se dedicaron al estudio de sus ideas, terminando por declararlas como modelo para la construcción de una escuela del trabajo soviética integrada y politécnica. ${ }^{8}$ En la década de 1920 , Dewey se convirtió en el pedagogo occidental de mayor popularidad en la Rusia soviética, algo que no resultaba del todo obvio, si se tiene en vista la actitud crítica del régimen hacia los modelos "burgueses." ¿Cómo puede explicarse tal situación?

Una razón puede verse en el hecho de que ciertas posiciones básicas de Dewey (que eran reflejo del pragmatismo norteamericano) podían asociarse con el marxismo. Entre ellas se cuentan: el tomar la praxis como fundamento de todo conocimiento y como único criterio de verdad válido; el acordar a la actividad práctico-material un espacio de privilegio en el desarrollo humano; el anular las diferencias de clase y el promover la justicia social; el reconocer el papel fundamental que la producción desempeńa en el desarrollo de la sociedad; el incluir a las masas populares en el control de la vida cultural, social y política. Todas estas ideas marxistas encontraban su paralelo en las de Dewey, quien abogaba también por un aprendizaje por medio de la experiencia y un trabajo práctico que se constituyera en la base del proceso de educación y formación; por una equiparación del trabajo físico con el intelectual, por una conexión de escuela y la producción, y por un vínculo estrecho entre la escuela y la vida social (cf. Dewey 1916/1993). El pedagogo marxista Pinkevič — cuya postura respecto de Dewey llegó a ser extremadamente crítica en la segunda itad de la década de 1920 — afirmó incluso que Dewey estaba "infinitamente más cerca de Marx y del comunismo ruso" que los pedagogos europeos occidentales, como Kerschensteiner, Lay, Scharrelmann, Forster o Seidel (Pinkevič 1925, p. 48; cf. 1930, p. 240).

Otra razón de la popularidad de Dewey era que los pedagogos soviéticos consideraban su idea de la "nueva" escuela como el modelo de escuela del trabajo más convincente. A diferencia de la escuela del trabajo de Kerschensteiner-de la que se criticaba, además de su carácter burgués, su desproporcionada orientación hacia las manualidades - Dewey aplicaba el principio del trabajo con el objetivo, ya no de desarrollar habilidades manuales concretas, sino de fomentar una formación integral del individuo. Por otra parte, subrayaba (al contrario que Kerschensteiner) la relevancia creciente que el desarrollo industrial ocupaba en la vida cultural de la sociedad y, por ende, en la educación y la formación (Dewey 1916/1993, p. 405ss., p. 412), elaborando, por último, un modelo de educación politécnica (cf. Klafki 1978, p. 789s.; cf. Dewey 1916/1993, pp. 406 y 408). Al mismo tiempo, Dewey reclamaba la igualdad de oportunidades educativas para todos los miembros de la sociedad. "Como pedagogo, Dewey era mucho más progresista que el educador de Múnich [i.e., Kerschensteiner]" (Pinkevič 1925, p. 59; 1930, p. 239s.). Por estos motivos, los pedagogos soviéticos terminaron por abandonar los modelos de reforma alemanes o 
europeos occidentales para aproximarse al modelo norteamericano. Si bien los orígenes de este viraje en la recepción de la pedagogía extranjera (que puede resumirse en la fórmula "Dewey en vez de Kerschensteiner") pueden encontrarse ya en la Rusia prerrevolucionaria (cf. Vencel en ACARE, F. 23, op. 1, ed. chr. 13; Levitin 1915; Ignat'ev/Odinetz/Novgorotsev 1933), éste sólo llegó a concretarse definitivamente en el período soviético.

Sin embargo, esta intensa recepción de Dewey en la Rusia soviética se debe también a una persona en particular: N. K. Krupskaja, esposa de V. I. Lenin y viceministra de Educación en el primer período soviético. Krupskaja tenía una actitud abierta hacia las propuestas de reforma reforma pedagógica originadas tanto en Rusia como en el extranjero. ${ }^{9}$ Entre las últimas, favorecía el sistema escolar estadounidense que, en su opinión, tenía un carácter mucho más democrático que la escuela alemana, la cual promovía el espíritu de sumisión. De acuerdo con Krupskaja, en los Estados Unidos el hijo del presidente de la nación compartía en la escuela el banco con el hijo del jornalero. En Alemania, por el contrario, la escuela estaba caracterizada "por una diferenciación de clases y estratos mucho más acentuada" (Krupskaja 1972, p. 331s.). ${ }^{10}$ Otros pedagogos soviéticos de importancia creían, al igual que Krupskaja, que el sistema escolar estadounidense no se caracterizaba por la diferenciación de clases sociales y se adaptaba con facilidad a los requerimientos que planteaba el desarrollo social y económico del estado, en activa colaboración con organizaciones públicas y bajo el control de la opinión pública. Más aún, muchos de ellos opinaban, curiosamente, que la implementación total de la escuela del trabajo y la vinculación de la misma con la producción era ya una realidad en los Estados Unidos (cf. Fortunatov en Rubinstein 1925, p. 64; Šackij 1928 en Rüttenauer/Schiff 1970, p. 386ss.; Lunačarskij 1924, p. 6). Dentro del panorama pedagógico que ofrecían los Estados Unidos, la concepción que resultaba más atrayente a los pedagogos soviéticos era la de Dewey.

\subsection{Los pedagogos liberales soviéticos y la recepción de Dewey (1917-1928)}

Tras haberse decidido en 1918 que la nueva escuela soviética debía ser una "escuela del trabajo," se suscitó de inmediato una intensa discusión acerca de la manera de ponerla en práctica. Existían concepciones muy variadas sobre lo que había que entender por una "verdadera" escuela del trabajo. En especial, los pedagogos soviéticos de tendencia liberal (Šackij, Fortunatov, Blonskij, Gerit, Gotalov-Gottlieb), así como también los pedagogos "estatales" abiertos a las ideas de reforma del extranjero (Krupskaja, Lunačarskij), se volvieron, en la primera mitad de la década de 1920, hacia la obra de Dewey, la cual, a su criterio, ofrecía el modelo más idóneo para la escuela del trabajo soviética. Este es un aspecto que pone de relieve, p. ej., Šackij, en un análisis de los distintos modelos de escuela del trabajo en Europa occidental y los Estados Unidos (Šackij 1918 en Rüttenauer/Schiff 1970, p. 264; cf. Zolotarev/ Golubev/Šackij 1918). En su opinión, la escuela del trabajo propuesta por Dewey constituía "el tipo de escuela más cercano ... al que venimos planeando para nuestra 
reforma escolar” (Šackij 1918 en Rüttenauer/Schiff 1970, p. 264; cf. Zolotarev/ Golubev/Šackij 1918). "Dewey ha sabido captar en su totalidad el espíritu de la nueva escuela, de la escuela que particularmente se necesita en Rusia" (Šackij en Dewey 1921, p. 6; cf. Gerit/Gotalov-Gottlieb 1925, p. 89). Fortunatov (1926, p. 13) escribe, por su parte, que el concepto de escuela del trabajo ofrecido por Dewey aporta una síntesis de todas la variantes de la escuela del trabajo provenientes de Europa occidental. Y Pinkevič, quien no dejaba de reconocer los logros pedagógicos de Dewey, si bien rechazaba a la vez su "ideología," escribe: "En las palabras de Dewey hay mucho de verdad y mucho de valioso, ya que vinculan la ciencia con la praxis, la vida y la producción. Hemos encontrado en ellas una serie de ideas que no podemos sino compartir" (Pinkevič 1930, p. 204s.). "El estudio de la obra de Dewey es un deber para todo pedagogo" (Pinkevič 1925, p. 64; cf. Šulgin 1918a, p. 6 y 1918 b, p. 15s.).

Al igual que lo habían hecho los partidarios de la "educación libre" antes de 1917, los pedagogos liberales soviéticos (algunos de los cuales también habían sido antes de 1917 seguidores de aquella corriente) efectuaron en la primera mitad de la década de 1920 una intensa recepción de las ideas de Dewey. En la obra de Dewey veían un programa pedagógico complejo, dentro del cual les resultaban de particular interés los siguientes reclamos básicos: 1) partir de la base del interés y del carácter del niño, renunciando a cualquier objetivo predeterminado que sirviera a un estado; 2) el aprendizaje por medio de la experiencia y el trabajo práctico autónomo; 3) la escuela como parte del entorno social. El fin último de los pedagogos liberales era el de construir, como lo planteaba Dewey, un sistema escolar que debía tanto promover el desarrollo individual como contribuir al bien de la comunidad democrática. Al igual que Dewey, consideraban que la escuela era el primer nivel de la sociedad democrática (Dewey 1916/1993, p. 409).

En especial Fortunatov, Blonskij, Šackij y Krupskaja ponían el acento en la propuesta de Dewey de conformar el proceso educativo "a partir de niño": sólo de tal modo podía lograrse una enseńanza exitosa. Krupskaja opinaba que Dewey había dejado sentado que "la individualidad del niño representaba la suma de ciertas fuerzas e instintos arraigados en el organismo" y que "tomando en consideración la individualidad del niño ... podían concretarse grandes logros” (Krupskaja 1919 en 1972, p. 331; cf. Blonskij 1924, p. 127; Fortunatov 1926, p. 13; Fortunatov en Rubinstein 1925, p. 73; Šackij 1922 en Rüttenauer/Schiff 1970, p. 283). Fortunatov, por su parte, consideraba especialmente revolucionaria la postura de Dewey de que el proceso educativo no tenía fin fuera de sí mismo: "El objetivo de la educación no puede ser la preparación para algo determinado. No, la educación debe aportar la posibilidad de un desarrollo integral adaptado a la individualidad del niño. ... Es imposible preparar al niño para una circunstancia determinada de su vida futura ..., ya que tales circunstancias pueden quedar superadas en el curso del tiempo. ... Por ello se deben desarrollar en el niño las capacidades que le permitan crear por sí mismo sus circunstancias de vida. ... La escuela absorbe influencias de la vida que la circunda y, a la vez, influye sobre la vida de la sociedad" (Fortunatov 1926, p. 73s.; cf. Šackij en Dewey 1921, p. 5). 
Por su lado, Šackij — uno de los partidarios más fervientes de Dewey en la Rusia soviética - señalaba que el mérito principal de Dewey consistía en haber reconocido el dualismo existente en las ciencias de la educación, el cual "se manifiesta en la división entre teoría y praxis, ciencia y vida, trabajo intelectual y trabajo manual” (Šackij en Dewey 1921, p. 5). Dewey había reconocido incluso "el dualismo existente en la sociedad de clases, que se refleja en los métodos y planes de estudio" (ibid.). La intención que guiaba a Dewey era la de establecer un sistema escolar que aboliera las disparidades sociales (ibid.). Šackij compartía con Dewey, además, la visión de que, aunque siempre se intentara por medio de la escuela consolidar las formas preexistentes de la vida social, la "misión de la educación" consistía en "promover los procesos de crecimiento dentro de la sociedad" (ibid.). Šackij conminaba a sus lectores rusos a "estudiar con la mayor atención" la obra de Dewey, "a sumergirse en cada una de las tesis del mejor filósofo contemporáneo" (ibid.).

Pero, en la Rusia soviética no sólo tuvo lugar una recepción de la teoría de Dewey: también se implementaron en la práctica algunos modelos de la pedagogía progresista. En 1925 dos pedagogos soviéticos de tendencia liberal, A. Gerit y A. GotalovGottlieb, presentaron la llamada "escuela del futuro," que Dewey describe en su libro del mismo nombre (Dewey/Dewey 1922d). Lo que estos educadores encontraban particularmente progresista en el planteamiento de de tales escuelas era, en primer lugar, que no perseguían objetivos utilitaristas, sino sólo pedagógicos y sociales; luego, que estaban pensadas para las masas y, por lo tanto, no se trataba de escuelas elitistas; por último, que se veían como modelos de sociedad democrática, de modo que contribuían a la paz social. La educación en estas escuelas estaba concebida de manera tal que unía de forma óptima los derechos del individuo con sus obligaciones sociales. Los pedagogos concluían que, por tanto, este tipo de educación podría ser aprovechado también en Rusia (cf. Gerit/Gotalov-Gottlieb 1925, p. 208).

Con estos antecedentes, no es de extrañar que la doctrina de Dewey haya sido utilizada activamente - sobre todo en la primera mitad de la década de 1920 — en la elaboración de un nuevo programa escolar. Los principales pedagogos "estatales" soviéticos hacían también público lo mucho que habían aprendido de Dewey. Por ejemplo, P. Blonskij — autor de La escuela del trabajo, libro muy popular no sólo en Rusia sino también en el mundo occidental — fue uno de los que mayor influencia de Dewey acusó. Declara Blonskij que, en la elaboración de su concepto de escuela del trabajo, tuvo "por única guía" a Dewey, Marx y la realidad rusa (Blonskij 1918/1973, p. 138). Más tarde habría de escribir: "De Dewey hemos aprendido a construir una escuela acorde con los requerimientos del nuevo siglo ... y a las necesidades e intereses del nińo" (Blonskij 1925, p. 213). E incluso el ministro de Educación admitió: "Mucho es lo que hemos aprendido de pedagogos como Dewey" (Lunačarski 1927 en 1984, p. 213). ${ }^{11}$

No obstante, en la intensa recepción de la obra de Dewey en la primera mitad de la década de 1920 los motivos exclusivamente pedagógicos tuvieron una incidencia mayor que los políticos. La doctrina de Dewey era tenida en cuenta por su valor pedagógico, por ser el mejor modelo de escuela que podía hallarse en la pedagogía internacional. Los textos de la época nos dejan la impresión de que el establecimiento 
de la escuela del trabajo soviética no habría sido posible en absoluto sin la orientación que brindaban los textos de Dewey. Esta presunción se ve apoyada por testimonios que dan cuenta de una intensa recepción de enfoques didáctico-metodológicos desarrollados sobre la base de la doctrina de Dewey. A ello dedicaremos la sección siguiente.

\subsection{El "método proyecto" y el plan Dalton al servicio de la escuela de masas soviética}

Después de que los pedagogos soviéticos hubieran acordado que el trabajo productivo debía ser el principio que guiase los contenidos de la nueva escuela, se planteó el interrogante acerca de qué formas y qué métodos de enseñanza serían los que harían posible la transmisión de dichos contenidos. En su opinión, lo más indicado a tal fin eran las metodologías didácticas que, partiendo más o menos de la pedagogía de Dewey, habían elaborado sus discípulos y seguidores (W.H. Kilpatrick, E. Parkhurst y E. Dewey). Según Krupskaja (en E. Dewey 1923, p. 8), estos enfoques didáctico-metodológicos abrían "horizontes completamente nuevos" a la escuela del trabajo soviética. Lo que más importaba a los pedagogos soviéticos era que estos métodos de enseńanza instruyeran a los alumnos en combinar de la mejor manera posible el trabajo individual con el colectivo y que permitieran, además, transmitir a los alumnos un conocimiento amplio del mundo, de modo que éstos pudieran organizar de forma correcta su propio trabajo y adquirir por sí mismo nuevos conocimientos (cf. Krupskaja en E. Dewey 1923, p. 11s.; Krupskaja 1922, pp. 163-168; Rubinstein 1925, p. 191s.; Pinkevič 1925, pp. 117 y 191ss.). En opinión de los pedagogos estatales, eran éstas cualificaciones de las que ningún ciudadano soviético podía prescindir.

En su prólogo a la traducción rusa del libro de E. Dewey The Dalton laboratory plan (1923), Krupskaja escribe: "Nos cuesta mucho a los rusos trabajar según un plan, evaluar las condiciones materiales ... El plan Dalton nos enseńa a trabajar de acuerdo con un plan, a calcular tiempos, fuerzas, posibilidades. En el momento actual nos es de extraordinaria importancia enseñar a los niños ... a organizar su trabajo. A este respecto el plan Dalton puede sernos de gran ayuda. Deberíamos someterlo a un detenido estudio" (Krupskaja en E. Dewey 1923, p. 11s.; cf. Krupskaja 1922, p. 165). M.M. Rubinstein, otro pedagogo soviético, se expresaba en términos similares: "El desarrollo de la capacidad de aprender de manera autónoma es un tema de gran actualidad, en especial para Rusia. Los campesinos y obreros rusos que han accedido al poder y asumido la tarea de construir un nuevo estado deben poder estar a la altura de sus responsabilidades dirigenciales" (Rubinstein 1925, p. 193). Y eran el plan Dalton y el "método proyecto" las metodologías más adecuadas para promover el desarrollo de las cualificaciones necesarias para ello.

Pinkevič veía en el plan Dalton otras ventajas adicionales: "Destruye los grupos pasivos en las clases ...; confiere al alumno una visión general del conjunto del trabajo realizado; le permite elegir por sí mismo sus tareas ...; elimina la coerción y otorga libertad al alumno ...; favorece el trabajo en la escuela, haciéndolo más flexible; crea 
condiciones óptimas para la cooperación entre docente y alumno" (Pinkevič 1925, p. 119s.). "Debe admitirse que el plan Dalton da respuestas a las demandas de la actividad, la motricidad y la dinámica infantiles" (id., p. 123). Era por ello que el plan Dalton y el método experimental debían estar, junto con el principio del trabajo, "entre los elementos básicos de una escuela socialista productiva" (id., p. 123). Krupskaja (en E. Dewey 1923, p. 7s.) afirma que es sólo por intermedio de tal metodología didáctica que puede crearse una escuela "nueva" que se distinga radicalmente de la antigua, con su disciplina de cuartel, su opresión de la personalidad y su enseñanza mecánica."

Pese a su aceptación casi unánime de los métodos didácticos "norteamericanos," los pedagogos soviéticos tenían también una visión crítica en cuanto a la medida en que éstos podían implementarse en la escuela del trabajo soviética. Por ejemplo, Pinkevič (1925, p. 120) señalaba las dificultades de llevar a la práctica el plan Dalton y el "método proyecto" dadas las "precarias condiciones materiales de la escuela soviética." Además, con vista a la implementación de estos métodos didácticos en Rusia, se propusieron algunos cambios que tenían como fin poner de relieve las intenciones del estado. Pinkevičc, por su parte, opinaba que podía correrse "el riesgo de que estos nuevos métodos fomenten en demasía los rasgos individualistas del carácter de los alumnos" (id., p. 121s.). Por ello, propuso que tanto el plan Dalton como el "método proyecto" se implementaran en Rusia con un mayor control sobre los alumnos y sus actividades. También debía concederse al trabajo colectivo un espacio más amplio del que los autores norteamericanos le asignaban. Rubinstein (1925, pp. 182, 196s. Y 199s.) reclamaba la adaptación del plan Dalton a la realidad soviética. Tenía que verse el plan dentro del contexto de la escuela de masas soviética: de otro modo, no valía la pena ocuparse de él. Puede decirse, entonces, que los principales pedagogos soviéticos favorecían la adopción del plan Dalton y el "método proyecto," pero bajo una forma sometida a la reflexión crítica y ajustada a la situación y objetivos politicos de Rusia.

La recepción de estas metodologías didácticas, más o menos elaboradas a partir de de la pedagogía de Dewey, se vio reflejada no sólo en trabajos científicos, sino también en documentos pedagógicos del Ministerio de Educación soviético. En 1923 el Consejo Científico del Estado (Gosudarstvennyj učenyj sovet) — en realidad, de su Sección Pedagógica bajo la dirección de Krupskaja — hizo públicos nuevos planes de estudio para la escuela del trabajo soviética, que lugo serían conocidos como "programa de los complejos" (kompleksnaja programma). En dichos planes se había tomado en consideración buena parte de las experiencias realizadas en las principales escuelas reformadas (por ejemplo, las escuelas experimentales de Šatursk y Gaginsk) (cf. Blonskij 1925, p. 8; Palčinskij 1928, p. 100). Antes de su aprobación por parte del ministerio, los nuevos planes fueron objeto de una intensa discusión que tuvo lugar en numerosas instituciones y congresos pedagógicos de las repúblicas soviéticas (cf. Blonskij 1925, p. 7s.). De este modo, los planes contemplaban no sólo las experiencias realizadas en las escuelas experimentales, sino también las conclusiones de la discusión pedagógica. No eran más que el resultado de la combinación de la doctrina marxista y las principales tendencias de reforma pedagógica rusas y del extranjero (cf. id., pp. 3-12). 
Los nuevos planes se caracterizaban por tres ideas centrales: "el trabajo productivo como núcleo alrededor del cual se concentra el resto de los materiales; clases integradas [llamadas 'complejos'] y una constante referencia de la educación a los problemas actuales" (Krupskaja en Anweiler 1978, p. 263s.). Era en vista de estos tres aspectos que se realizaba la selección y estructuración de los materiales didácticos en una clase determinada. Estos materiales estaban, por un lado, divididos según su contenido (en concordancia con la doctrina marxista) en tres campos: naturaleza, trabajo y sociedad; y, por otro, segmentados en distintos "complejos," de acuerdo con la concepción pedagógica de la enseñanza interdisciplinaria (cf. id., p. 264; Blonskij 1925, p. 7s.).

En la confección de estos nuevos planes colaboraron varios de los principales pedagogos soviéticos, quienes, a su vez, tomaban activa parte en la recepción de Dewey (por ejemplo, Krupskaja, Blonskij, Šackij, Šulgin, Pinkevič). Muchos fueron los autores que pusieron de relieve la conexión existente entre los nuevos planes y las ideas pedagógicas de Dewey, así como con las metodologías didácticas basadas en ella (cf. Ignat'ev 1925, p. 11; Zakožhurnikova en Ignat'ev 1925, pp. 145-150; Blonskij 1925, pp. 3-12; Merzon 1925, p. 129ss.; Levin 1925, p. 87; Palčinskij 1928, p. 117; Rubinstein 1925, p. 204). Además, los métodos norteamericanos - recomendados por las más altas instancias educativas de la URSS - fueron puestos en práctica en la escuela soviética de la primera mitad de la década de 1920, como lo testimonian numerosos trabajos pedagógicos (cf. Moiseev/Beskin 1927; Finkel 1925; Gencov/ Dzens-Litovskij et al. 1925; Šulgin 1925a, pp. 125-127; Šackij 1925; Ignat'ev 1925; Levin 1925; Palčinskij 1928, p. 117; Rubinstein 1925, p. 204). A esas alturas, la influencia ejercida por la obra de Dewey en la política educativa y en la vida escolar de la Rusia soviética era imposible de ignorar. Todavía no se veía en el origen "burgués" de tal doctrina un impedimento para su aplicación en la escuela de masas de la URSS. Esta situación habría de cambiar en la segunda mitad de la década.

\subsection{La escuela al servicio del partido Bolchevique: el rechazo a la pedagogía de Dewey}

Con la creciente "intensificación de la lucha de clases” y la acentuación del control del partido Bolchevique sobre la escuela - en particular a partir de 1928 - los criterios políticos e ideológicos pasaron a dominar la evaluación de las ideas pedagógicas. En consecuencia, las concepciones de Dewey, que no contenían los principios marxistasleninistas de "lucha de clases"o "dictadura del proletariado," fueron objeto de una crítica creciente durante la segunda mitad de la década de 1920. Curiosamente, esta crítica provenía, en parte, de los mismos pedagogos que, a principios del decenio, habían abogado por la adopción activa de tales ideas en la Rusia soviética.

Tras 1925, en especial Šulgin, Kamenev y Pinkevič comenzaron a pronunciarse de manera cada vez más reprobatoria respecto de las enseñanzas de Dewey. El punto central de su crítica era que Dewey no había acertado a establecer objetivos educativos concretos (cf. Šulgin 1925b, p. 15s.). Dewey no daba respuesta a un interrogante en el que los pedagogos soviéticos tenían particular interés: para qué clase social se 
educaba a los jóvenes. En opinión de Šulgin y Pinkevič, lo único importante para Dewey era crear un entorno que favoreciera el crecimiento del niño (cf. Pinkevič 1930, p. 241; Šulgin 1925b, p. 16). Esto no se compadecía con la visión de los pedagogos soviéticos, pues para ellos "lo primero" eran "las tareas dictadas por el estado, y sólo después la particularidades del desarrollo infantil” (Kamenev 1925, p. 334).

Desde la perspectiva de los pedagogos marxistas, Dewey había evitado la cuestión de la lucha de clases. Kamenev (1925, p. 134) censuraba a Dewey por no querer cambiar el sistema social existente. Šulgin, por su parte, escribía que a Dewey sólo le interesaba compensar los conflictos sociales, "estrangular la conciencia de clase, justificar la democracia, adaptar la escuela a la sociedad burguesa.... [Al hacer esto] está traicionando a los jóvenes trabajadores, pretendiendo separarlos de la clase obrera a la que pertenecen" (Šulgin 1925b, p. 106ss.). "Pretende crear trabajadores obedientes que sientan aprecio por la burguesía y que crean que su propio trabajo es un beneficio para la sociedad" (Kamenev 1925, p. 118). En opinión de Pinkevič, "la intención de Dewey es reconciliar la clase obrera con su condición de oprimida. Sobre este tipo de demócratas o socialistas se advierte ya en el Manifiesto comunista, que pretenden que los obreros sigan viviendo en una sociedad burguesa, pero poniendo su odio de lado" (Pinkevič 1925, p. 60). Partiendo de tales críticas, sólo había que dar un paso para afirmar: "La filosofía de Dewey ... se orienta al pasado, no tiene futuro. ... La pedagogía de Dewey es la pedagogía del imperialismo" (Šulgin 1925b, p. 110s.; cf. Medynskij 1929, p. 320s.; Pinkevič 1930, p. 241).

La posición de estos pedagogos marxistas respecto de la cuestión de los objetivos de la educación encontró su expresión más clara en las palabras de Šulgin:
"Siempre hay objetivos en la educación: eso es un hecho. Por ello, haríamos mejor en discutir no sobre si se deben plantear objetivos o no, sino sobre cuáles son los objetivos que debemos plantear a las escuelas. Me parece, camaradas, que hoy es más importante que nunca hablar sobre los objetivos de la educa- ción. ... Debemos preparar a los jóvenes que serán reemplazo de nuestra gene- ración. ... Los niños deben participar del trabajo ... en nombre ... de las organi- zaciones de adultos. ... Los niños deben acercarse a la clase obrera y campesina, adquirir su ideología. ... Debemos formar luchadores por los ideales de la clase obrera ... y constructores de una sociedad nueva. En ello consiste nuestra tarea" (Šulgin 1925b, pp. 18-25).

También las metodologías didácticas elaboradas sobre a base de la pedagogía de Dewey (el plan Dalton y el "método proyecto") fueron sometidas a una dura crítica. Mientras que a comienzos de la década de 1920 estos métodos habían sido saludados por los pedagogos soviéticos, lo que ahora se decía era que el plan Dalton y el "método proyecto" eran sólo una versión mejorada de la "formación libresca pasiva" y que no llevaban a la creación de una comunidad, sino al desarrollo del espíritu de competencia. La imitación de tales metodologías equivaldría a cultivar la escuela burguesa en la Rusia soviética. (cf. Kamenev 1925, p. 337).

En una mirada retrospectiva, puede decirse que la crítica de los pedagogos 
soviéticos había malinterpretado lo que en verdad era una idea revolucionaria en la pedagogía de Dewey. Al negarse a formular objetivos externos a la educación y al declarar que "el proceso educativo no tiene otro fin más que sí mismo," (Dewey 1916/1993, p. 75), Dewey ofrecía al problema fundamental de la teoría pedagógica una solución que era tan sencilla como genial: Dewey rechaza tanto la idea de un "desarrollo" completamente libre, que excluye toda influencia sobre el desarrollo del niño, como la idea de una "preparación" o "influencia” que no tome en consideración las experiencias del nińo (cf. Dewey 1916/1993, pp. 75-113, p. 147; cf. Oelkers en Dewey 1993, p. 504). Dewey no pretende formar un ciudadano "útil" que sea garante de la persistencia de un sistema estatal en particular. No define objetivo determinado alguno que deba alcanzarse. Para Dewey, lo "único" que cuenta es la acumulación sostenida de episodios de aprendizaje y experiencias reconstruibles (cf. Dewey 1916/1993, pp. 108, 137).

Sin embargo, esta idea de Dewey resultaba incompatible con las concepciones de la pedagogía soviética, ya que ésta orientaba sus objetivos - a diferencia de Dewey — no al individuo, sino a los intereses políticos de un partido. Los intereses del estado se antepusieron a los intereses del individuo y la escuela pasó a estar al servicio del estado: precisamentelo que Dewey intentaba combatir en su pedagogía. Era claro que Dewey no podía servir a los fines del partido Bolchevique, y por ello se lo eliminó, a finales de la década de 1920, de la discusión en materia de política escolar.

\section{Conclusiones}

Los comienzos de la recepción de la pedagogía de Dewey en Rusia fueron relativamente tardíos. Esto puede deberse, en parte, a las dificultades en las comunicaciones con la lejana Norteamérica. Ello no obstante, puede atribuirse un peso mayor a la tradición de recepción que imperaba en Rusia antes de 1917, la cual se orientaba preponderantemente hacia modelos alemanes. En la Rusia presocialista los responsables de la recepción de Dewey fueron en particular los partidarios de la "educación libre" y los exponentes de la "formación realista." En 1915 las ideas de Dewey encontraron acceso a los planes de reforma del Ministerio de Educación ruso. Así, Dewey, pese a la baja intensidad - al menos en términos cuantitativos - de su recepción, pasó a cobrar una influencia en el sistema escolar ruso equivalente a la de Kerschensteiner, el pedagogo más popular en la Rusia presocialista.

En vísperas de la Revolución de Octubre comenzó a delinearse en Rusia una nueva tendencia en la recepción de la pedagogía extranjera. Los reformistas rusos fueron alejándose de los modelos europeos occidentales - en especial alemanes - para orientarse cada vez más hacia las concepciones de Dewey. Desde el punto de vista de los reformistas rusos, Dewey reunía en sí distintos reclamos de reforma, sin caer en los múltiples errores de los pedagogos europeos occidentales. Pero, por otra parte, este viraje tenía también una causa política: Alemania se encontraba en guerra con Rusia desde 1914, lo que repecutió de manera negativa en las relaciones 
germano-rusas en el terreno de la pedagogía. Con los Estados Unidos, en cambio, no sólo no había guerra: el país, con su orden social democrático y su sistema escolar organizado según lineamientos básicamente democráticos, concitaba, como modelo a imitar, un creciente interés en Rusia - un país en el que, por aquel entonces, se acentuaban los conflictos sociales y se encontraba al borde de la revolución social. Seguramente la recepción de Dewey se vio beneficiada en cierto sentido por tales circunstancias.

Ya en el período soviético, la recepción de Dewey experimentó un enorme auge. Las tesis de Dewey fueron objeto de intenso estudio por parte de los principales pedagogos estales rusos en la primera mitad de la década de 1920, y se las incluyó en la planificación de la escuela del trabajo soviética. La pedagogía de Dewey y las metodologías derivadas de ésta encontraron su reflejo en los planes de estudio elaborados por el Ministerio de Educación en 1923, que se llevaron efectivamente a práctica en la escuela de masas soviética. Esto se debía, por una parte, a que la pedagogía pragmática de Dewey era, entre todos los enfoques reformistas, el que mejor se podía compatibilizar con las ideas básicas del marxismo. Pero, por otra parte, se debía también a la excelencia misma de los razonamientos pedagógicos de Dewey. Y se debía, además, en útima instancia, a los principales pedagogos y políticos educativos liberales de la Rusia soviética, quienes - movidos en principio por razones pragmáticas - apoyaron la recepción y adopción de modelos de reforma pedagógica extranjeros.

Con la acentuación de la dictadura del partido Bolchevique en todos los campos de la cultura, las ideas de Dewey, al igual que muchas otras, fueron objeto de una crítica creciente. Dicha crítica se orientaba menos a aspectos concretos de su pedagogía que a su "falata" de intencionalidad política. La pedagogía de Dewey no podia ponerse la servicio de ideas tales como las de "lucha de clases," "dictadura del proletariado" o "comunismo," que tanto importaban a los pedagogos soviéticos. Y ello porque Dewey ponía en primer plano al individuo y su autonomía, y no los intereses de un estado o de un movimiento político.

Entre Dewey y sus críticos soviéticos mediaba también una concepción diferente de la relación entre fines y medios. Una de las características más conspicuas del experimento social llevado a cabo en la Rusia soviética es la enorme discrepancia entre los altos fines perseguidos por los nuevos gobernantes y los métodos empleados para alcanzarlos. Se pretendía construir el socialismo - es decir, un orden social justo - pero se lo hizo apelando a medios autoritarios, dictatoriales, inhumanos. Esta particularidad del desarrollo social influyó en gran medida en la política escolar de la Rusia soviética y, en consecuencia, en la recepción de Dewey. Mientras que para Dewey la libertad es, a la vez, fin y medio del cambio social (Dewey 1916/1993, pp. 137-151; Bohnsack 1976, p. 402), los bolcheviques pretendieron imponer la idea de libertad social por medio de métodos autoritarios, incluso en la escuela, haciendo caer así en el descrédito la idea que pensaban imponer. Y la pedagogía de Dewey era difícil de conciliar con el sistema educativo de un estado autoritario. 


\section{Bibliografía}

I. Material de archivo

(a) Materiales del Archivo Científico de la Academia Rusa de Educación (ACARE), Moscú. F. (fond [fondo]) 23, op. (opis' [registro]) 1, ed. chr. (edinica chranenija [ítem conservado] 13. = Archivo personal de N. K. Vencel. Incluye memorias de los trabajos realizados entre 1912 y 1915.

(b) Materiales del Archivo Histórico Estatal (AHE) de San Petersburgo F 740, op. 10, Nr. 14. = "P. N. Ignat'ev, ministro de Educación. Su desempeño." F 1099, op. 1, Nr. 13. = Fondo de A. A. Klopov. "Sobre el nombramiento de P. N. Ignat'ev como ministro.” Semblanza de P. N. Ignat'ev (17/01/1915).

\section{Fuentes impresas: Diarios de sesiones de congresos, protocolos, resoluciones,} informes anuales, escritos oficiales y otros documentos

Dnevik pervogo Vserossijkogo s-ezda po voprosam narodnogo obrazovanija [Diario de sesiones del Primer Congreso Panruso de Educación Popular], Ulej, San Petersburgo, 1913.

Doklady, prenija i postanovlenija vtoroj sekcii pervogo Vserossijskogo s-ezda po narodnomu obrazovaniju [Diario de sesiones, debates y resoluciones del Primer Congreso Panruso de Educación Popular], Ulej, San Petersburgo, 1913.

KPSS v rezolucijach s-ezdov, konferencij i plenumov CK [Partido Comunista de la Unión Soviética, en resoluciones de reuniones, conferencias y sesiones plenarias del Comité Central], t. 2, Moscú, 1954.

Pervyj Vserossijskij s-ezd po proveščeniju: Zasedanie 26-go avgusta 1918 [Primer Congreso Panruso de Educación. Sesión del 26 de agosto de 1918], s.l., s.e., s.d.

Otčet o dejatel'nosti pedagogičeskogo obščestva [Informe sobre las actividades de la Sociedad de Pedagogía], s.e., Moscú, 1899.

Položhenie o edinoj trudovoj škole Rossijskoj Socialističeskoj Federativnoj Sowetskoj Respubliki [Reglamento de la escuela integrada del trabajo de la República Socialista Federativa Soviética de Rusia], Vserossijskij Central'nyj Ispolnitel'nyj komitet, Narkompros po prosveščeniju, Moscú, 1918.

Rezoljucija Pervogo Vserossijskogo s-ezda po voprosam narodnogo obrazovanija s 22 dekabrja 1913 po 3 janvarja 1914 goda [Resolución del Primer Congreso Panruso de Educación Popular del 22 de diciembre de 1913 al 3 de enero de 1914], Kommerčeskaja skoropečatnja, San Petersburgo, 1914.

III.

O. Anweiler, Geschichte der Schule und Pädagogik in Rußland vom Ende des Zarenreiches bis zum Beginn der Stalin-Ära (Berlín, 1978).

P.P. Blonskij, Pedagogika [Pedagogía] (Moscú: Rabotnik Prosveščenija, 1924).

P.P. Blonskij, Novye Programmy GUS'a učitel' [Nuevos programas del Consejo Científico del Estado y de los docentes] (Moscú: Rabotnik Prosveščenija, 1925).

P.P. Blonskij, Die Arbeitschule [La escuela del trabajo] (Paderborn, 1918/1973).

F. Bohnsack, Erziehung zur Demokratie. John Deweys Pädagogik und ihre Bedeuting für die Reform unserer Schule (Ravensburg, 1976).

N.V. Čechov, Svobodnaja škola. Opyt organizacii školy novogo tipa [Una nueva escuela. Experiencia de organización de una escuela superior de nuevo tipo] (Moscú : I.D. Sytin, 1907).

E. Dewey, Daltonskij laboratornyj plan [El plan experimental Dalton]. Trad. de R. Langsberg, prólogo de N.K. (Moscú: Krupskaja. Novaya Moskva, 1923).

J. Dewey, Škola i obščestvo [The School and Society] (Moscú: I.D. Sytin, 1907).

J. Dewey, Psichologia i pedagogika myšlenija [How we think] (Moscú: Mir, 1915). 
J. Dewey y E. Dewey, Školy budǔ̌čego [Schools of Tomorrow]. Trad. de R. Langsberg. En: Svobodnoe vospitanie [La Educación Libre] (1916/1917) 1: 21-48; 2: 55-74; 3: 21-42; 4/5: 17-40; 6: 19-34; 7/8: 25-40; 9/10: 67-94; 11/12: 21-40.

J. Dewey, Vvedenie v filosofiju vospitanija [Democracy and Education: An Introduction to the Philosophy of Education]. Prólogo de S.T. Šackij. (Moscú: Rabotnik Prosveščenija, 1921).

J. Dewey, Psichologija i pedagogika myšlenija [How we think]. Trad. de N.M. Nikol'skaja, red. por N.D. Vinogradov, Gosizdat RSFSR, Berlín (1922a).

J. Dewey, Škola i obščestvo [The School and Society] (Moscú: Rabotnik Prosveščenija, $1922 b)$.

J. Dewey, Škola i rebenok [The Child and the Curriculum]. Trad. de L. Azarevič. (Moscú: Gosizdat, 1922c).

J. Dewey y E. Dewey, Školy budǔ̌čego [Schools of Tomorrow]. Trad. de R. Langsberg. (Moscú: Rabotnik Prosveščenija, 1922d).

J. Dewey, Škola i rebenok [The Child and the Curriculum]. Trad. de L. Azarevič (Moscúl Petrogrado: Gosizdat, 1923).

J. Dewey, Skola i obščestvo [The School and Society] (Moscú: Gosizdat, 1924).

J. Dewey, Škola i obščstvo [The School and Society]. Trad. de G.A. Lučinskij (Moscú: Rabotnik Prosveščenija, 1925).

J. Dewey, Demokratie und Erziehung. Eine Einleitung in die philosophische Pädagogik (Weinheim/Basel, 1916/1993).

I. Finkel, Daltonplan v škole fabzavuča: Sbornik statej i materialov [El plan Dalton en las escuelas fábrica. Recopilación de artículos y materiales] (Moscú: Novaja Moskva, 1925).

A. Fortunatov, Teorija trudovoj školy v ee istoričeskom razvitii [La teoría de la escuela del trabajo en su desarrollo histórico], t. 1 (Moscú: Mir, 1926).

L. Froese, Ideengeschichtliche Triebkräfte der russischen und sowjetischen Pädagogik. (Heidelberg, 1963).

F. Gansberg, "Nauka v narodnoj škole" [Wissenschaft in der Volksschule/La ciencia en la escuela popular]. Trad. de V. Koševič. En: Svobodnoe vospitanie [La Educación Libre] 8 (1907/1908), 73-92.

I.A. Gencov, A.I. Dzens-Litovskij, y N.V. Kazmin, N.V.: Dal'tonplan na rabfake [El plan Dalton en las facultades obreras] (Leningrado: Priboj, 1925).

A. Gerit y A. Gotalov-Gottlieb, Sovremennye pedagogičeskie tečenija [Corrientes actuales de la pedagogía]. Gosudarstvennoe izdatel'stvo Ukrainy (Cherson, 1925).

B.V. Ignat'ev, Dal'tonplan i novejšie tečenija russkoj pedagogičeskoj mysli [El plan Dalton y las más actuales corrientes de la pedagogía en Rusia] (Moscú: Mir, 1925).

P.N. Ignat'ev, D.M Odinetz, y P.J. Novgorotsev, Russian Schools and Universities in the World War (New Haven: Yale University Press, 1929).

W.H.E. Johnson, Russia's Educational Heritage Nueva York: Ostagon Books, 1969).

S.A. Kamenev, Sovetskaja trudovaja škola [La escuela del trabajo soviética] (Rostov del Don: Sevkavkniga, 1925).

P.F. Kapterev, “Organizacija avtonomnoj školy” [La organización de las escuelas autónomas]. En: Pedagogičeskaja mysl' [Pensamiento pedagógico] 9/12 (1918), 147-49.

W. Klafki, "Die Aktualität der Pädagogik von John Dewey." En: Zeitschrift für Pädagogik 24 (1978): 781-93.

B.B. Komarovskij, Pedagogika Dewey [La pedagogía de Dewey], t. 1. s.e. (Bakú, 1926).

N.A. Konstantinov, Očerki po istorii srednej skoly [Apuntes para una historia de la escuela superior] (Moscú: Učpedgiz, 1947).

N.K. Krupskaja, "Evelyn Dewey. Dal'tonskij plan laboratornoj raboty v škole. Helen Parkhurst. Vospitanie pri pomošči Dal'tonskogo plana" [Evelyn Dewey. The Dalton laboratory plan. Helen Parkhurst. Education on the Dalton plan]. En: Na putjach $k$ novoj škole [En camino a una nueva escuela] 2/3 (1922), 163-68. 
N. K. Krupskaja, "Metod proektov v politprosvetrabote” [El 'método proyecto' en el trabajo de formación política]. En: Kommunističeskoe Prosveščenie [Formación Comunista] 3/9 (1923): 25-31.

N.K. Krupskaja, Sozialistische Pädagogik, t. 1 (Berlín, 1972).

L. Levin, Novye puti školnoj raboty (metod proektov) [Nuevos caminos del trabajo escolar (el 'método proyecto')] (Moscú: Rabotnik Prosveščenija, 1925).

S.A. Levitin, "Trudovaja škola — škola buduščego" [Escuela del trabajo-escuela del futuro]. En: : Svobodnoe vospitanie [La Educación Libre] 4 (1912/1913): 23-32.

S.A. Levitin, "Trudovoj prinzip vospitanija. Doklady, čitannye na Vserossijskom s-ezde po narodnomu obrazovaniju [El principio del trabajo en la educación. Ponencias presentadas en el Primer Congreso Panruso de Educación Popular]. En: Svobodnoe vospitanie [La Educación Libre] 3 (1914/1915): 25-40.

S.A. Levitin, "Trudovaja škola i professionalizm” [La escuela del trabajo y el profesionalismo]. En: Russkaja škola [La Escuela Rusa] 4 (1915): 71-89.

Levitin, S.A.: Trudovaja škola — škola buduščego [Escuela del trabajo-escuela del futuro]. Praktičeskie znania, Moscú 1916.

A.V. Lunačarskij, Na novych putjach [Por nuevos caminos] (Samara: Gubizdat, 1924).

A.V. Lunačarskij, “Die Ziele der Sowjetschule.” En: Kommission für deutsche Erziehungsund Schulgeschichte der Akademie der Pädagogischen Wissenschaften der DDR (ed.): Monumenta Paedagogica. Dokumente und Materialien zu den deutsch.sowjetischen Beziehungen auf bildungspolitischem und pädagogischem Gebiet 1917-1933. t. XXII (Berlín, 1984), 210-15.

J. Mamontov, Chrestomatija sovremennych pedagogičeskich tečenij [Crestomatía de las actuales corrientes pedagógicas] (Ekaterinoslav: Gosudarstvennoe Izdatel'stvo Ukrainy, 1924).

I. Mchitarjan, Der russische Blick auf die deutsche Reformpädagogik. Zur Rezeption deutscher Schulreformideen in Rußland zwischen 1900 und 1917 (Hamburgo, 1998).

E.D. Medynskij, "Pedagogičeskie tečenija v Rossii konca 19-načala 20 veka" [Las corrientes pedagógicas en Rusia hacia finales del siglo XIX y comienzos del XX]. En: A.G. Kalašnikov (ed.), Pedagogičeska enciklopedija [Enciclopedia de la pedagogía], t. 1 (Moscú: Rabotnik Prosveščenija, 1927), 392-95.

E.D. Medynskij, Istorija pedagogiki v svjazi s ekonomičeskim razvitiem ob̌̌čestva [Historia de la pedagogía y su relación con el desarrollo económico de la sociedad], t. 2 (Moscú: Rabotnik Prosveščenija, 1929).

E.D. Medynskij, Istorija russkoj pedagogiki do Velikoj Oktjabr'skoj socialističeskoj revoljucii [Historia de la pedagogía rusa hasta la Gran Revolución Socialista de Octubre] (Moscú: Gosučpedgiz narkomprosa RSFSR, 1938).

Merzon (no consta el nombre de pila del autor), "Opyt primenenija Dal'ton-plana v trudovoj škole” [La aplicación del plan Dalton en la escuela del trabajo]. En: Narodnoe Prosvešcenie [La Educación Popular] 10/11 (1925): 128-32.

L.V. Moiseev y M.A. Beskin, M.A. Dal'tonplan v školach učeničestva. Opyt raboty Moskovskoj školy učeničestva [El plan Dalton en las escuelas vocacionales. Experiencia de trabajo en la Escuela Vocacional de Moscú] (Moscú: Transpečat' NKPS, 1927).

A. Pabst, "Praktičeskoe vospitanie" [Educación práctica]. Trad. de V. Koševič. En: Svobodnoe vospitanie [La Educación Libre] 1 (1909/1910): 63-88.

V.O. Palčinskij, Protiv kompleksnoj sistemy i o metodach prepodavanija voobšče [Contra el sistema de complejos y sobre los métodos de enseñanza en general]. Kabinet NOT pri sekcii gigieny Odesskoj naučno-issledovatel'skoj kafedry profelaktičeskoj mediciny (Odesa, 1928).

A.P. Pinkevič, Pedagogika. trudovaja škola [Pedagogía. La escuela del trabajo], t.2 (Moscú: Rabotnik Prosveenija, 1925).

A.P. Pinkevič, Kratkij očerk istorii pedagogiki [Breves apuntes para la historia de la pedagogía] (Járkov: Proletarijj, 1930). 
"Reforma srednej školy" [Reforma de la escuela superior] (autor desconocido). En: Russkaja skola [La Escuela Rusa] 5/6 (1915): 78-85.

M.M. Rubinstein, Trudovaja škola v svete istorii i sovremennosti [La escuela del trabajo en la historia y la actualidad] (Leningrado, Sejatel', 1925).

I. Rüttenauer y B. Schiff, S. T. Šackij: Ausgewählte pädagogische Schriften (Heidelberg, 1970).

S.T. Šackij, "Detskij trud i novye puti” [El trabajo infantil y los nuevos caminos]. En: Svobodnoe vospitanie [La Educación Libre] 6 (1907/1908): 47-56.

S.T. Šackij, A.U. Zelenko y N.J. Kazimirov, Deti-rabotniki buduščego [Los niños: trabajadores del mañana]. s.e., Moscú.

V.N. Šulgin, "Detskaja kommuna” [La comuna infantil]. En: Narodnoe prosveščenie [La Educación Popular] 23/25 (1918a): 6.

V.N. Šulgin, “Školy buduščego" [Escuelas del futuro] de J. Dewey y E. Dewey. En: Svobodnoe vospitanie [La Educación Libre] 19 (1918b): 15-16.

V.N. Šulgin, “Obščestvennaja rabota v škole” [El trabajo público en la escuela]. En: Narodnoe prosvešcenie [La Educación Popular] 10/11 (1925a): 125-27.

V.N. Šulgin, Osnovnye voprosy social'nogo vospitanija [Problemas fundamentales de la educación social] (Moscú: Rabotnik Prosveščenija, 1925 b).

I.S. Simonov y N.V. Čechov, Dal'ton-plan v russkoj škole [El plan Dalton en la escuela rusa] (Leningrado: Brockhaus-Ethron, 1924).

P. Sokolov, Istorija pedagogičeskich sistem [Historia de los sistemas pedagógicos] (San Petersburgo: O.V. Bogdanova, 1913).

K.N. Vencel, Bor'ba za svobodnuju školu [La lucha por una escuela libre] (Moscú: A.P. Pečkovskogo, 1906).

K.N. Vencel, Kak sozdat' svobodnuju školu (Dom svobodnogo rebenka) [Cómo se funda una escuela libre (Casa del Niño Libre)] (Moscú: I.N. Kušnerev i Kº 1908).

K.N. Vencel, "Ideal'naja škola buduščego i spobosy ee osuščestvlenija" [La escuela ideal del futuro y los medios para su realización]. En: Svobodnoe vospitanie [La Educación Libre] 8/9 (1908/1909): 1-28 y 19-44.

K.N. Vencel, "Otdelenie školy ot gosudarstva” [La separación de la escuela y el estado]. En: Svobodnoe vospitanie [La Educación Libre] 6 (1916/1917): 1-8.

K.N. Vencel, "Sovremenny moment i svobodnoe vospitanie" [El momento actual y la educación libre]. En: Svobodnoe vospitanie [La Educación Libre] 1-3 (1918): 13-18.

S.A. Zolotarev, A.V. Golubev y S.T. Šackij, Trudovaja škola [La escuela del trabajo] (Moscú: K.L Menšova, 1918).

\section{Notas}

1. Una versión en inglés del presente artículo apareció en Studies in Philosophy and Education 19 (2000).

2. El proceso de recepción puede reconstruirse mediante fuentes originales, es decir, a través de las distintas aportaciones de autores rusos acerca de Dewey y su pedagogía. Sin embargo, los motivos y la significación de esta recepción sólo pueden analizarse por medio del contexto de la evolución social y político-escolar de Rusia. Este contexto de recepción será tratado a través de la bibliografía secundaria correspondiente.

3. En este congreso, organizado por el Comité de Instrucción Elemental de San Petersburgo, tomaron parte 6.500 pedagogos (cf. Medynskij 1927, p. 394; 1938, p. 441ss.; "Dnevik pervogo Vserossijkogo s-ezda po voprosam narodnogo obrazovanija" 1913). El encuentro, bajo la dirección del pedagogo "estatal" Mamontov fue transformándose en un foro del movimiento reformista, con el riesgo constante de ser disuelto por las autoridades. 
4. Cf. asimismo "Dnevik pervogo Vserossijskogo s-ezda po voprosam narodnogo obrazovanija” 1913; "Doklady prenija i postanovlenija vtoroj sekcii pervogo Vserossijskogo s-ezda po narodnomu obrazovaniju” 1915.

5. "Rezoljucija Pervogo Vserossijskogo s-ezda po voprosam narodnogo obrazovanija s 22 dekabrja 1913 po 3 janvarja 1914 goda” 1914, p. 19.

6. Ignat'ev había sido, entre 1904 y 1907, presidente del zemstva de Kiev (los zemstvas eran órganos de autoadministración en las zonas rurales). Entre 1907 y 1909 ocupó el cargo de gobernador de Kiev. En 1909 pasó al ministerio de Agricultura, donde se desempeńó como jefe de departamento hasta 1914. El 9 de enero de 1915 fue nombrado ministro de Educación de Rusia. Según la opinión de sus contemporáneos, Ignat'ev era una persona "competente, accesible, correcta y humilde," que tenía la capacidad de adaptarse muy velozmente a las tareas que se le asignaran (AHE F. 740, op. 10, Nr. 14 y F 1099, op. 1, Nr. 13).

7. En la Crestomatía de las actuales corrientes pedagógicas, editada por Mamontov (Mamontov 1924), se publicaron pasajes de los trabajos de Dewey Škola i ob̌̌čestvo [The School and Society] y Vvedenie v filosofiju vospitanija [Democracy and Education: An Introduction to the Philosophy of Education].

8. Cf. "Položhenie o edinoj trudovoj škole Rossijskoj Socialističeskoj Federativnoj Sowetskoj Respubliki” 1918.

9. Krupskaja había realizado, ya antes de 1917, diversos estudios acerca de los sistemas educativos y las tendencias de reforma pedagógica en los Estados Unidos y Europa occidental. Además, publicaba aportaciones en el más radical de los periódicos pedagógicos de la Rusia prerrevolucionaria, La Educación Libre (Svobodnoe vospitanie), manifestando el aprecio que sentía por el movimiento reformista ruso, en especial por la pedagogía de Tolstoj, si bien, en ciertos puntos, mantenía una posición muy crítica respecto de ésta.

10. Cf. asimismo "Pervyj Vserossijskij s-ezd po proveščeniju: Zasedanie 26-go avgusta 1918 " s/d, p. 1-5.

11. Cf. "Položhenie o edinoj trudovoj škole Rossijskoj Socialističeskoj Federativnoj Sowetskoj Respubliki” 1918, p. 5. 